\title{
CAMERA AUTOCALIBRATION AND THE CALIBRATION PENCIL
}

\author{
ANTONIO VALDÉS AND JOSÉ IGNACIO RONDA
}

\begin{abstract}
We study the geometric object given by the set of lines incident with the absolute conic. We see that this object is given by a pencil of quadrics of $\mathbf{P}^{5}$, which is characterized. We describe some of its most relevant properties for the camera autocalibration problem. Finally, we illustrate the applicability of the theory proposing a linear algorithm for the metric upgrading of a projective calibration of a set of ten or more cameras with varying parameters and known skew and aspect ratio.
\end{abstract}

\section{INTRODUCTION}

Camera autocalibration is possible due to the existence of the absolute conic lying in the plane at infinity [5]. After its introduction a great amount of ideas flourished for recovering the Euclidean structure of space and the camera intrinsic parameters from a projective calibration of the scene (see [1], [3] for recent accounts of the techniques).

The dual absolute quadric [10] represented another important source of ideas for algorithms. This geometrical object is given by the set of planes tangent to the absolute conic, thus encoding at the same time the plane at infinity and the absolute conic. The estimation of the dual quadric is often simpler (see, e.g. [1], [3], [8] and the references therein). But as natural as considering the tangent planes to the absolute conic is to consider the lines intersecting it, which provides another way to encode the Euclidean structure of space.

The aim of this work is to study, with a view on the autocalibration problem, some of the relevant properties of this entity. This object is seen to be a pencil of quadrics of $\mathbf{P}^{5}$, defined by the Klein quadric $\Omega$ and a rank-three quadric $\Sigma$ whose characterization we provide. We will call this pencil the calibration pencil. We will see how Euclidean properties are translated to this setting and can be obtained from it.

The calibration pencil allows for the use of linear estimations of the Euclidean structure in certain scenarios in which it was not possible to do it with either the absolute conic or the dual absolute quadric. As an application, we propose a linear least-squares algorithm which provides the camera parameters from a minimal set of ten cameras, assuming known pixel shape and unknown and varying focal length and principal point.

A relevant precedent of this work is [6] where, from a different motivation, a linear algorithm for the autocalibration of a set of twenty cameras is presented that is based on the matrix of the quadric $\Sigma$, also providing an almost complete algebraic characterization of this matrix.

Date: March 10, 2004. 
The paper is organized as follows. Section 2 summarizes the standard projective camera model and establishes some notations. Section 3 provides for the reader's convenience a summary of the properties of Plücker coordinates that are relevant for our purposes. Section 4 introduces the calibration pencil, which is characterized in Section 5. Section 6 provides procedures to extract from the calibration pencil the information of interest. Finally, Section 7 illustrates the application of the theory with an algorithm for the linear autocalibration of a set of cameras.

\section{CAMERA MOdel}

In this paper we assume that the camera is modeled by the equation $\mathbf{q} \sim P \mathbf{Q}$, where $\sim$ means equality up to an homogeneous non-zero factor, $\mathbf{Q}=(x, y, z, t)^{T}$ denotes the homogeneous coordinates of a spatial point and $\mathbf{q}=(u, v, w)^{T}$ denotes the homogeneous image coordinates. The matrix $P$ is a $3 \times 4$ matrix given by $P=K(R \mid-R \mathbf{t})$, where here the matrix $K$, called calibration matrix, is a $3 \times 3$ matrix given by

$$
K=\left(\begin{array}{ccc}
\alpha_{u} & -\alpha_{u} \cot \theta & u_{0} \\
0 & \alpha_{v} / \sin \theta & v_{0} \\
0 & 0 & 1
\end{array}\right)
$$

being $u_{0}$ and $v_{0}$ the non-homogeneous coordinates of the principal point, $\alpha_{u}$ and $\alpha_{v}$ are the pixel scale factors and $\theta$ is the skew angle between the axes of the pixel coordinates. We denote by $\tau$ the pixel aspect ratio given by $\tau=\alpha_{u} / \alpha_{v}$. The matrix $R$ is a rotation matrix which gives the camera orientation, and $\mathbf{t}$ are the coordinates of the camera center (see [3] as a general reference on this model).

We recall here that it is possible to obtain a projective calibration only from image correspondences. This means that, given a set of $N$ cameras, $N \geq 2$, we can obtain a set of calibration equations $\mathbf{q}_{i} \sim \hat{P}_{i} \hat{\mathbf{Q}}$, where $\hat{P}_{i}=P_{i} H^{-1}$ and $\hat{\mathbf{Q}}=H \mathbf{Q}$, $H$ being a non-singular $4 \times 4$ matrix which gives a coordinate change from the Euclidean coordinates to some arbitrary projective coordinates.

Euclidean calibration can be defined as the obtainment of a matrix $H$ changing the projective coordinates of a given projective calibration to some Euclidean coordinate system. This can be done, in particular, if the absolute conic or some equivalent geometrical object is identified in the given coordinate system.

We will denote by $(U, V, W)^{T}$ a set of Euclidean coordinates adapted to the camera, so the actual retinal coordinates $\mathbf{u}^{T}=(u, v, w)^{T}$ are related with $\mathbf{U}^{T}=$ $(U, V, W)^{T}$ by the equation $\mathbf{u}^{T} \sim K \mathbf{U}^{T}$.

\section{PlǘCKer COORdinates}

We next summarize some properties of the Plücker coordinates that will be used in the following. A general reference for this topic is [7].

The set of all the lines of the projective space $\mathbf{P}^{3}$ is called the Grassmannian $\operatorname{Grass}(3,1)$. Given two different points of $\mathbf{P}^{3}, \mathbf{Q}=\left(x_{0}, x_{1}, x_{2}, x_{3}\right)$ and $\mathbf{Q}^{\prime}=$ $\left(x_{0}^{\prime}, x_{1}^{\prime}, x_{2}^{\prime}, x_{3}^{\prime}\right)$, we define the Plücker coordinates of the line $\mathbf{Q} \mathbf{Q}^{\prime}$ as the set of numbers $p_{i j}=x_{i} x_{j}^{\prime}-x_{i}^{\prime} x_{j}$. Due to the antisymmetry $p_{i j}=-p_{j i}$, we can restrict ourselves to consider the six numbers $p_{01}, p_{02}, p_{03}, p_{12}, p_{13}, p_{23}$. This construction allows to consider $\operatorname{Grass}(3,1)$ as a subset of $\mathbf{P}^{5}$. Expanding the identity $\operatorname{det}\left(\mathbf{Q}, \mathbf{Q}^{\prime}, \mathbf{Q}, \mathbf{Q}^{\prime}\right)=0$ in terms of Plücker coordinates, we obtain the quadratic relation $\Omega=p_{01} p_{23}+p_{02} p_{31}+p_{03} p_{12}=0$. It can be checked that any point of the 
so called Klein quadric $\Omega \subset \mathbf{P}^{5}$ corresponds exactly with a line in $\mathbf{P}^{3}$ (by abuse of notation, we will use the same term for the quadric as a set of points, its matrix and its polynomial).

We remind that two points $r$ and $s$ are conjugate with respect to a quadric $Q$ if $r^{T} Q s=0$. This means that each of them lies in the polar hyperplane of the other. Thus, if $Q$ is regular, we can assign to each point $r$ the hyperplane of its conjugate points, of coordinates $Q r$, obtaining the so-called polarity mapping $Q: \mathbf{P}^{5} \rightarrow \mathbf{P}^{5^{\star}}$. Its inverse mapping is called the dual polarity mapping $Q^{\star}: \mathbf{P}^{5^{\star}} \rightarrow \mathbf{P}^{5}$.

Conjugacy with respect to Klein quadric has a nice geometrical interpretation. Two lines of Plücker coordinates $r=\left(p_{i j}\right)_{i<j}$ and $r^{\prime}=\left(p_{i j}^{\prime}\right)_{i<j}$ intersect if and only if they are conjugate with respect to the Klein quadric, i.e., $r^{T} \Omega r^{\prime}=0$. Consequently, the mapping $\mathbf{P}^{5} \supset \Omega \ni r \mapsto \Omega r \in \mathbf{P}^{5 \star}$ which assigns to $r$ its polar tangent hyperplane of coordinates $\Omega r$ can be interpreted as a correspondence assigning to a line the set of all its incident lines $\Omega r \cap \Omega$.

A change of coordinates $x_{i}^{\prime}=\sum_{j=0}^{3} a_{i}^{j} x_{j}$ in $\mathbf{P}^{3}$ induces a corresponding linear change of coordinates in $\mathbf{P}^{5}$ as follows:

$$
p_{i j}^{\prime}=\sum_{k, l=0}^{3}\left(a_{i}^{k} a_{j}^{l}-a_{j}^{k} a_{i}^{l}\right) p_{k l},
$$

as can be shown by a direct computation. Of course, this change of coordinates leaves invariant the Klein quadric.

We call a projective coordinate system on $\mathbf{P}^{5}$ allowable when it comes from a projective coordinate system of $\mathbf{P}^{3}$. From now on this will be the only kind of coordinate systems we will use in $\mathbf{P}^{5}$.

Equation (2) produces an embedding of the group $\mathbf{P G L}(4, \mathbf{C})$ of projective transformations of $\mathbf{P}^{3}$ into the group $\mathbf{P G L}(6, \mathbf{C})$ of projective transformations of $\mathbf{P}^{5}$. We will denote by $G$ the image of this embedding. Moreover, $G$ lies inside the group $\operatorname{Aut}(\Omega)$ of projective transformations of $\mathbf{P}^{5}$ leaving invariant the Klein quadric $\Omega$, constituting a subgroup of index 2. Being $\Omega$ a non-degenerate quadric, Aut $(\Omega)$ is conjugate to a projective complex orthogonal group, i.e., there exists a regular matrix $M$ such that $M^{-1} \operatorname{Aut}(\Omega) M$ is the complex orthogonal group. For these results and further information on this topic, the reader can consult, for example, [2, page 122$]$.

Alternatively, we can consider a line as defined by intersection of the planes of coordinates $u$ and $v$. This line has dual Plücker coordinates $\pi_{i j}=u_{i} v_{j}-u_{j} v_{i}$. These coordinates are related to ordinary Plücker coordinates by the formulas (see [7, page 246]):

$$
\frac{\pi_{23}}{p_{01}}=\frac{\pi_{31}}{p_{02}}=\frac{\pi_{12}}{p_{03}}=\frac{\pi_{01}}{p_{23}}=\frac{\pi_{02}}{p_{31}}=\frac{\pi_{03}}{p_{12}} .
$$

As is well known, any regular quadric in $\mathbf{P}^{3}$ contains two one-parametric families of complex lines. Analogously, the Klein quadric, as any regular quadric in $\mathbf{P}^{5}$ contains two three-parametric families of two-dimensional subspaces, which have a very interesting interpretation in terms of the geometry of $\mathbf{P}^{3}$ [7, page 392]. These families of planes are called $\alpha$-planes and $\beta$-planes. The set of points in a given $\alpha$-plane correspond to a star of lines in $\mathbf{P}^{3}$, i.e., the set of lines incident with a given point of space, while the points of a $\beta$-plane correspond to the set of lines of $\mathbf{P}^{3}$ contained in some plane of space. Finally, a line of $\mathbf{P}^{5}$ contained in the Klein 
quadric can be identified with a pencil of lines, i.e., the set of lines in a given plane which are also incident with some point. From this interpretation it is immediate that two $\alpha$-planes or two $\beta$-planes intersect in a single point of $\mathbf{P}^{5}$ and that an $\alpha$-plane and a $\beta$-plane either do not intersect or intersect in a line of $\mathbf{P}^{5}$.

\section{The Calibration Pencil}

Although for the sake of clarity all the results of this section are stated for an arbitrary non-degenerate conic $C$ of $\mathbf{P}^{3}$, the reader should have in mind that they will be applied to the absolute conic lying in the plane at infinity.

The next Theorem establishes that the lines intersecting the conic $C$ are given, in Plücker coordinates, by a pencil of quadrics in $\mathbf{P}^{5}$. In the case that the conic is the absolute conic this pencil will be called the calibration pencil. We will see in this section that it encodes all the neccesary information to perform an affine and Euclidean calibration.

Theorem 4.1. The lines intersecting a non-degenerate conic in $\mathbf{P}^{3}$ are given by the intersection of the Klein quadric $\Omega$ with a rank-three quadric $\Sigma$ in $\mathbf{P}^{5}$.

Proof. Using a projective change of coordinates, if necessary, we can assume that the conic is given by

$$
\left.\begin{array}{rl}
x_{0}^{2}+x_{1}^{2}+x_{2}^{2} & =0 \\
x_{3} & =0
\end{array}\right\}
$$

Since the intersection of a line of dual Plücker coordinates $\left(\pi_{i j}\right)$ with the plane $x_{3}=0$ is the point $\left(\pi_{12}, \pi_{20}, \pi_{01}, 0\right)$, those lines intersecting the conic are given by

$$
\Sigma: \pi_{01}^{2}+\pi_{12}^{2}+\pi_{20}^{2}=0,
$$

which is the equation of a rank-three quadric in $\mathbf{P}^{5}$. This, together with the equation of the Klein quadric

$$
\Omega: \pi_{01} \pi_{23}+\pi_{02} \pi_{31}+\pi_{03} \pi_{12}=0
$$

constitute the necessary and sufficient conditions for the $\pi_{i j}$ to be the coordinates of a line intersecting the conic.

As suggested by equations (5) and (6), we will use from now on the coordinate system in $\mathbf{P}^{5}$ qiven by the vector of dual Plücker coefficients $\left(\pi_{01}, \pi_{12}, \pi_{20}, \pi_{13}, \pi_{03}, \pi_{23}\right)$ which according to equation (3) is proportional to the vector $\left(p_{23}, p_{03}, p_{13}, p_{20}, p_{12}, p_{01}\right)$, so that the matrices of $\Sigma$ and $\Omega$ will have the simple forms

$$
\Sigma=\left(\begin{array}{llllll}
1 & 0 & 0 & 0 & 0 & 0 \\
0 & 1 & 0 & 0 & 0 & 0 \\
0 & 0 & 1 & 0 & 0 & 0 \\
0 & 0 & 0 & 0 & 0 & 0 \\
0 & 0 & 0 & 0 & 0 & 0 \\
0 & 0 & 0 & 0 & 0 & 0
\end{array}\right), \Omega=\left(\begin{array}{llllll}
0 & 0 & 0 & 0 & 0 & 1 \\
0 & 0 & 0 & 0 & 1 & 0 \\
0 & 0 & 0 & 1 & 0 & 0 \\
0 & 0 & 1 & 0 & 0 & 0 \\
0 & 1 & 0 & 0 & 0 & 0 \\
1 & 0 & 0 & 0 & 0 & 0
\end{array}\right) .
$$

It is worth to note here that, since $\Omega$ and $\Sigma$ intersect transversally, the set of quadrics passing through this intersection is given by the pencil $\Omega+\lambda \Sigma$ (see, for example, [2, page 301]), so we can identify the intersection locus $\Omega \cap \Sigma$ with the pencil. 


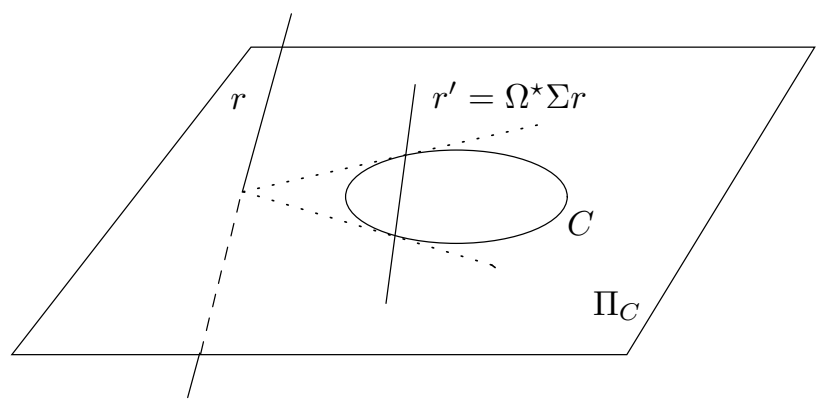

FiguRE 1. Interpretation of the mapping $r \mapsto \Omega^{\star} \Sigma r$. If $C$ is the absolute conic, given a line $r, \Sigma r$ are the coordinates of a hyperplane of $\mathbf{P}^{5}$ whose intersection with the Klein quadric gives the set of all the lines orthogonal to $r$. These lines are those that intersect the plane at infinity at $r^{\prime}$, so that $r^{\prime}=\Omega^{\star} \Sigma r$.

A better understanding of the geometrical meaning of the matrix $\Sigma$ results from realizing that it establishes orthogonality in the case that the conic is the absolute conic. This is the content of the next Theorem.

Theorem 4.2. Let $r, r^{\prime} \in \operatorname{Grass}(3,1)$ be two lines in $\mathbf{P}^{3}$ and $p, p^{\prime}$ their points of intersection with a plane $\Pi_{C}$ containing a non-degenerate conic $C$. Then $p$ and $p^{\prime}$ are conjugate with respect to $C$ if and only if $r$ and $r^{\prime}$ are conjugate with respect to the quadric $\Sigma \subset \mathbf{P}^{5}$ associated to $C$ given in the previous Theorem.

Proof. Let us use a projective coordinate system adapted to the conic, as in the proof of Theorem 4.1, so the conic $C$ has the equations showed in (4). If the lines $r$ and $r^{\prime}$ have Plücker coordinates $\left(\pi_{i j}\right)$ and $\left(\pi_{i j}^{\prime}\right)$, respectively, their intersections with the plane where the conic lies are the points of coordinates $p=$ $\left(\pi_{12}, \pi_{20}, \pi_{01}, 0\right)$ and $p^{\prime}=\left(\pi_{12}^{\prime}, \pi_{20}^{\prime}, \pi_{01}^{\prime}, 0\right)$, which are conjugate with respect to $C$ if and only if $\pi_{12} \pi_{12}^{\prime}+\pi_{20} \pi_{20}^{\prime}+\pi_{01} \pi_{01}^{\prime}=0$, which is equivalent to $r^{\prime T} \Sigma r=0$ where $r=\left(\pi_{01}, \pi_{12}, \pi_{20}, \pi_{13}, \pi_{03}, \pi_{23}\right)^{T}$ and $r^{\prime}=\left(\pi_{01}^{\prime}, \pi_{12}^{\prime}, \pi_{20}^{\prime}, \pi_{13}^{\prime}, \pi_{03}^{\prime}, \pi_{23}^{\prime}\right)^{T}$.

Let us denote also by $\Pi_{C}$ the $\beta$-plane associated to the plane of the conic $C$. If $C$ is the absolute conic, the restricted polarity mapping

$$
\mathbf{P}^{5} \supset \Omega \backslash \Pi_{C} \ni r \mapsto \Sigma r \in \mathbf{P}^{5 \star}
$$

can be interpreted as the mapping which assigns to each line represented by $r \in \Omega$ the set of all its orthogonal (although not necessarily incident) lines $\Sigma r \cap \Omega$. If $r$ belongs to the plane at infinity then this mapping is not defined since $\Pi_{C}$ is the projective space $\mathbf{P}(\operatorname{ker} \Sigma)$ associated to the vector space ker $\Sigma$. We can also consider the restricted composite mapping $\Omega^{\star} \Sigma$,

$$
\mathbf{P}^{5} \supset \Omega \backslash \Pi_{C} \stackrel{\Sigma}{\longrightarrow} \mathbf{P}^{5 \star} \stackrel{\Omega^{\star}}{\longrightarrow} \mathbf{P}^{5}
$$

where $\Omega^{\star}: \mathbf{P}^{5 \star} \rightarrow \mathbf{P}^{5}$ is the dual polarity induced by $\Omega$. To understand this composite mapping, let us first recall that if $u$ is a tangent hyperplane to the Klein quadric at $r \in \Omega$ then $\Omega^{\star} u=r$ and $u \cap \Omega$ represents the set of lines incident with $r$. Therefore, given $r \in \Omega \backslash \Pi_{C}, \Omega^{\star} \Sigma r$ is just the line of $\Pi_{C}$ representing the set of orthogonal directions to $r$. Consequently, $\Sigma \Omega^{\star} \Sigma$ vanishes on any vector 
representing a line $r \in \Omega \backslash \Pi_{C}$, and therefore everywhere. This property will prove to be important in the sequel.

Note that $\Omega^{\star}$ is represented by the adjoint matrix of $\Omega$, which in any allowable coordinate system is given by the same matrix $\Omega$ given by (7). The distinction between $\Omega$ and $\Omega^{\star}$ will be maintained to avoid a loss of geometrical meaning.

Finally observe that, since $r$ and $r^{\prime}$ intersect if and only if $r^{T} \Omega r=0$, the two lines are simultaneously intersecting and orthogonal if and only if they are conjugate with respect to the whole pencil of quadrics spanned by $\Omega$ and $\Sigma$.

\section{Characterization of the Calibration pencils associated With CONICS}

In order to refine initial estimates of a candidate $\Sigma$ obtained by linear methods it is convenient to have a characterization of the space of possible $\Sigma$ matrices. We study such a characterization in the following theorems.

Theorem 5.1. Let $\mathbf{P}(\operatorname{Sym}(6, \mathbf{C}))$ be the set of $6 \times 6$ symmetric matrices defined up to non-zero multiples, which can be identified with the quadrics of $\mathbf{P}^{5}$. The set $\mathrm{S}_{0} \subset \mathbf{P}(\operatorname{Sym}(6, \mathbf{C}))$ of those quadrics associated with non-degenerate conics of $\mathbf{P}^{3}$ is an open dense subset of the eight-dimensional irreducible algebraic variety

$$
\mathbf{S}=\{\Sigma \in \mathbf{P}(\operatorname{Sym}(6, \mathbf{C})): \operatorname{rank}(\Sigma) \leq 3 \text { and } \operatorname{ker} \Sigma \text { contains a } \beta \text {-plane }\}
$$

given by those matrices whose rank is exactly three. Moreover, the $\beta$-plane in the kernel of each $\Sigma \in \mathrm{S}_{0}$ corresponds to the plane of the conic in $\mathbf{P}^{3}$.

Proof. To see that $\mathrm{S}$ is an algebraic variety of dimension eight we use a standard technique (see [2, chapter 10]). Let us consider the Grassmanian $\operatorname{Grass}_{\beta}(5,2)$ given by the $\beta$-planes of $\mathbf{P}^{5}$, which is an irreducible three-dimensional variety, since each $\beta$-plane corresponds to a plane of $\mathbf{P}^{3}$ and therefore is isomorph to $\mathbf{P}^{3}$. Let us denote by $\mathrm{T}$ the variety of symmetric matrices of order six having rank three or less. We define the variety $\Phi \subset \mathrm{T} \times \operatorname{Grass}_{\beta}(5,2)$ given by $\Phi=\{(\Sigma, \Lambda): \Lambda \subset \operatorname{ker} \Sigma\}$. Let us see that the fiber of the projection onto the second factor has dimension five. We consider a fixed $\beta$-plane $\Lambda$. Using a coordinate change $A \in G$ which makes the plane associated to $\Lambda$ the plane $x_{3}=0$, we can assume that $\Lambda$ has equations $\pi_{01}=\pi_{12}=\pi_{20}=0$, so $\Sigma$ has the form

$$
\Sigma=A^{T}\left(\begin{array}{cc}
H & 0 \\
0 & 0
\end{array}\right) A
$$

where $H \neq 0$ is a symmetric $3 \times 3$ matrix. So clearly the generic fiber of the projection onto the second factor is an irreducible variety of dimension five, since so is the variety of such matrices $H$. From this it follows that $\Phi$ is an irreducible variety of dimension $\operatorname{dim} \operatorname{Grass}_{\beta}(5,2)+5=8$.

Finally note that $S$ is the projection of $\Phi$ onto the first factor, and that this projection is generically injective. From this it follows that $S$ is an irreducible algebraic variety of the same dimension that $\Phi$.

The set $S_{0}$ consisting of the matrices of $S$ of rank exactly three is an open Zariski subset of $\mathrm{S}$, so dense in S.

To see that the $\beta$-plane in which the kernel of a matrix $\Sigma \in \mathrm{S}_{0}$ consists is the plane of the associated conic, consider the conic given, after the coordinate change 
$A$, by $x_{3}=0$ and the equation

$$
\left(\begin{array}{lll}
x_{2} & x_{0} & x_{1}
\end{array}\right) H\left(\begin{array}{l}
x_{2} \\
x_{0} \\
x_{1}
\end{array}\right)=0 .
$$

It is immediate to check that the equation of the lines in $\mathbf{P}^{3}$ intersecting $C$ is given by the quadric of matrix $\Sigma$ of equation (8).

Theorem 5.2. (cf. [6]) Let $\Sigma$ be a quadric of $\mathbf{P}^{5}$. The following conditions are equivalent

(1) $\Sigma \Omega^{\star} \Sigma=0$ and $\operatorname{rank} \Sigma=3$.

(2) The image of $\Omega^{\star} \Sigma$ is a plane contained in $\Omega$ and therefore an $\alpha$ or $\beta$-plane.

(3) The projective subspace associated to the kernel of $\Sigma$ is a plane contained in $\Omega$.

Proof. 1) $\Longleftrightarrow$ 2) Let us take $\rho \in \mathbf{P}^{5}$ and not in the kernel of $\Sigma$. The condition $\Omega^{\star} \Sigma \rho \in \Omega$ is equivalent to

$$
\left(\Omega^{\star} \Sigma \rho\right)^{T} \Omega\left(\Omega^{\star} \Sigma \rho\right)=0 \Longleftrightarrow \rho^{T} \Sigma^{T} \Omega^{\star T} \Omega \Omega^{\star} \Sigma \rho=0 \Longleftrightarrow \rho^{T} \Sigma^{T} \Omega^{\star} \Sigma \rho=0
$$

This proves that the condition $\Sigma \Omega^{\star} \Sigma=0$ is equivalent to the fact that the image of $\Omega^{\star} \Sigma$ is contained in $\Omega$. On the other hand, $\operatorname{rank} \Sigma=3$ is equivalent to the fact that the vector dimension of the image of $\Omega^{\star} \Sigma$ is three, and conversely.

1) $\Longrightarrow 3$ It is immediate the equivalence of the conditions $\Sigma \Omega^{\star} \Sigma=0$ and $\operatorname{im}\left(\Omega^{\star} \Sigma\right) \subset \operatorname{ker} \Sigma$, independently of any rank assumption. Since $\operatorname{rank} \Sigma=3$ and $\Omega$ is a full-rank quadric both linear subspaces have the same dimension and therefore must coincide.

$3) \Longrightarrow 1$ ) Being the projective space associated to ker $\Sigma$ a plane, we conclude that $\Sigma$ must be of rank three. Being a plane contained in the Klein quadric, it must be an $\alpha$ or $\beta$-plane. Let us assume that it is an $\alpha$-plane, being the other case treated analogously. We can use an allowable coordinate system of $\mathbf{P}^{5}$ such that this $\alpha$-plane corresponds to the star through the point of coordinates $(0,0,0,1)$, so the $\alpha$-plane has Plücker equations $\pi_{13}=\pi_{03}=\pi_{23}=0$. This implies that $\Sigma$ has a matrix of the form $\left(\begin{array}{cc}0 & 0 \\ 0 & K\end{array}\right)$. From this it follows by a direct computation that $\Sigma \Omega^{\star} \Sigma=0$.

The following Corollary is a direct consequence of the previous Theorems.

Corollary 5.3. A quadric $\Sigma \in \mathbf{P}(\operatorname{Sym}(6, \mathbf{C}))$ belongs to $\mathrm{S}_{0}$ if and only if the image of $\Omega^{*} \Sigma$ expand a $\beta$-plane.

Observe that if a symmetric matrix $\Sigma$ has rank three and verifies $\Sigma \Omega^{\star} \Sigma=0$ then the image of $\Omega^{*} \Sigma$ is necessarily either an $\alpha$-plane or a $\beta$-plane, and only in the last case it belongs to $S_{0}$. Therefore this is a discrete condition, which can be easily checked using Lemma 8.1.

It is possible to propose some invariant conditions which allow us to identify the quadric $\Sigma$ within the pencil. To this purpose we need the following Theorem. Given a square matrix $M$, we denote by $\operatorname{tr}(M)$ the trace of $M$ and by $\hat{\operatorname{tr}}(M)$ the antitrace, i.e., the sum of the elements of the antidiagonal.

Theorem 5.4. The condition of having antitrace zero is preserved by the group of transformations preserving $\Omega$. 
Proof. Let us take a matrix $A$ such that $A^{t} \Omega A \sim \Omega$ and a square matrix $H$ of order six having null antitrace. Since $\Omega \Omega=I$ we have that $\Omega A^{t} \sim A^{-1} \Omega$. Therefore

$$
\hat{\operatorname{tr}}\left(A^{t} H A\right)=\operatorname{tr}\left(\Omega A^{t} H A\right) \sim \operatorname{tr}\left(A^{-1} \Omega H A\right)=\operatorname{tr}(\Omega H)=\hat{\operatorname{tr}}(H)=0 .
$$

Corollary 5.5. (cf. [6]) Given any matrix $\Sigma^{\prime}$ of the calibration pencil different from $\Omega$, the rank three matrix of the pencil $\Sigma \in \mathrm{S}_{0}$ is given by $\Sigma=\Sigma^{\prime}-\frac{1}{6} \hat{t r}\left(\Sigma^{\prime}\right) \Omega$.

Proof. Since $\hat{\operatorname{tr}}(\Sigma)=0$, the results follows from the previous Theorem.

\section{EXtRACTING INFORMATION FROM THE CALIBRATION PENCIL}

6.1. Obtainment of the plane at infinity. In the absence of noise it is possible to obtain the plane of the conic as the plane of $\mathbf{P}^{3}$ associated to the $\beta$-plane given by the kernel of $\Sigma$. However, if $\Sigma$ is estimated from noisy data there is not guarantee that this kernel will be a $\beta$-plane. Now we introduce an object associated to the calibration pencil which will allow us to obtain the plane of the conic directly as a plane of $\mathbf{P}^{3}$. Given any quadric $\Sigma_{\lambda}=\Sigma+\lambda \Omega$ of the pencil of quadrics of Theorem 4.1 and a generic line defined by a pair of planes of coordinates $u$ and $v$, we can obtain a symmetric biquadratic form $S(u, v)$ just by substituting each $\pi_{i j}$ by $u_{i} v_{j}-u_{j} v_{i}$ in the equation of the quadric associated to $\Sigma_{\lambda}$. This has the effect of restricting $\Sigma_{\lambda}$ to the Klein quadric, so the biquadratic form $S(u, v)$ is determined uniquely (up to multiples) by the pencil, and does not depend on the particular element chosen, as long as it is not the Klein quadric ${ }^{1}$.

We define the null space of $S(u, v)$ as $\operatorname{Null}(S)=\left\{u \in \mathbf{C}^{4}: S(u, v)=0 \forall v \in \mathbf{C}^{4}\right\}$.

Theorem 6.1. The null space of the symmetric biquadratic form $S(u, v)$ is the one-dimensional space given by the coordinates of the plane of the conic.

Proof. Using a coordinate system adapted to the conic, a direct computation shows that

$$
S(u, v) \sim\left(u_{0} v_{0}+u_{1} v_{1}+u_{2} v_{2}\right)^{2}-\left(u_{0}^{2}+u_{1}^{2}+u_{2}^{2}\right)\left(v_{0}^{2}+v_{1}^{2}+v_{2}^{2}\right)=0
$$

which immediately gives that $\operatorname{Null}(S)=(0,0,0,1)$, which are the coordinates of the plane of the conic.

Note that the obtainment of the plane at infinity from $S$ is a linear least-squares problem.

6.2. Obtainment of the camera intrinsic parameters. Let us see how $\Sigma$ also encodes the internal parameters of each camera. But first we need the following technical result. We recall here that a homography $h: \mathbf{P}^{n} \rightarrow \mathbf{P}^{n}$ is a projective mapping induced by a non-singular $(n+1) \times(n+1)$ matrix.

Lemma 6.2. The restriction of the embedding $\Phi: \operatorname{Grass}(3,1) \hookrightarrow \mathbf{P}^{5}$ to any star of lines or to the lines of any plane of $\mathbf{P}^{3}$ is a homography.

Proof. Let us consider the case of a star of lines through a point $P_{0} \in \mathbf{P}^{3}$. The case of a plane of lines follows from this first case by duality. We write the homography $\Phi$ using point-Plücker coordinates. Take a projective reference $\mathfrak{R}=$ $\left\{\mathbf{X}_{0}, \mathbf{X}_{1}, \mathbf{X}_{2}, \mathbf{X}_{3}, \mathbf{E}\right\}$ such that $\mathbf{X}_{3}=P_{0}$. Given any line $r \in \operatorname{Star}\left(P_{0}\right)$, we can

\footnotetext{
${ }^{1}$ The biquadratic form $S$ is just the Chow point associated with $C$ [2].
} 
compute its Plücker coordinates using the points $P_{0}=(0,0,0,1)$ and $P=r \cap$ Plane $\left(\mathbf{X}_{1} \mathbf{X}_{2} \mathbf{X}_{3}\right)=\left(x_{0}, x_{1}, x_{2}, 0\right)$, thus obtaining the coordinates of

$$
\Phi(r) \sim\left(x_{2}, x_{0}, x_{1}, 0,0,0\right) .
$$

This makes apparent that $\Phi$ is indeed a homography between $\operatorname{Star}\left(P_{0}\right)$ and $\Pi_{P_{0}}$.

The next Theorem will provide us with a straightforward way to obtain the projection of the absolute conic onto a retinal plane.

Theorem 6.3. Let us consider in $\mathbf{P}^{3}$ a non-degenerate conic $C$ lying in a plane $\Pi$ and the star of lines through a point $P \notin \Pi$. The intersection of the pencil $\Sigma+\lambda \Omega$ associated to the conic with the $\alpha$-plane corresponding to $\operatorname{Star}(P)$ is a conic in $\mathbf{P}^{5}$ representing the cone defined by $C$ and $P$.

Proof. Since $\Phi(\operatorname{Star}(P))$ lies in $\Omega$, the restriction of the pencil $\Sigma+\lambda \Omega$ to $\Phi(\operatorname{Star}(P))$ is the single conic $\sigma=\Sigma \cap \Phi(\operatorname{Star}(P))$ and not a proper pencil of conics.

Now there are two conics defined in the projective plane $\operatorname{Star}(P)$, that defined by $C$ and $P$ and that given by $\Phi^{-1}(\sigma)$. Using Theorem 4.2 together with the previous Lemma, it is easily seen that the two conics define the same conjugacy relation, and so they coincide.

\section{Algorithms}

A detailed study of the algorithms that can be designed from the theory above falls beyond the intended scope of this work. We will content ourselves here just by proposing a linear method for finding the plane at infinity and the intrinsic parameters of the set of cameras.

In [6] a linear algorithm is given to calibrate a set of twenty or more cameras assuming zero skew. The use of the theory developed above permits to design an algorithm to obtain by linear methods an Euclidean calibration from a projective calibration of 10 or more cameras assuming that the aspect ratios $\tau_{k}$ and the skew angles $\theta_{k}$ of the cameras are known.

The absolute conic intersects the retinal plane in two points, which are called the cyclic points at infinity of the plane. In the Euclidean coordinate system associated to the camera its coordinates are $(U, V, W) \sim(1, \pm i, 0)$, where $i=\sqrt{-1}$. Therefore its retinal coordinates are

$$
\left(\begin{array}{ccc}
\alpha_{u} & -\alpha_{u} \cot \theta & u_{0} \\
0 & \alpha_{v} / \sin \theta & v_{0} \\
0 & 0 & 1
\end{array}\right)\left(\begin{array}{l}
1 \\
i \\
0
\end{array}\right)=\left(\begin{array}{c}
\alpha_{u}-\alpha_{u} \cot \theta i \\
\alpha_{v} i / \sin \theta \\
0
\end{array}\right) \sim\left(\begin{array}{c}
\tau e^{-i \theta} \\
1 \\
0
\end{array}\right) .
$$

This means that back-projecting these points we obtain a pair of lines for each camera which will intersect the absolute conic. Now the problem consists in recovering the plane at infinity and the absolute conic from this data.

\subsection{Algorithm.}

(1) Back-project the points $I_{k}=\left(\tau_{k} e^{-i \theta_{k}}, 1,0\right)$ and $\bar{I}_{k}=\left(\tau_{k} e^{i \theta_{k}}, 1,0\right), k=$ $1, \ldots, N$, obtaining the lines $r_{k}=\hat{P}_{k}^{-1}\left(I_{k}\right)$ and $\bar{r}_{k}=\hat{P}_{k}^{-1}\left(\bar{I}_{k}\right)$. Compute the Plücker coordinates of the lines $r_{k}, \bar{r}_{k}$.

(2) Obtain the pencil of quadrics in $\mathbf{P}^{5}$ through these points by means of a linear least-squares method. 
(3) Compute the biquadratic symmetric form $S(u, v)$, as indicated in Theorem 6.1. Calculate the coordinates of the plane at infinity $\Pi_{\infty}$ as the null space of $S$.

(4) Obtain for each camera the projected absolute conic using Theorem 6.3. Obtain from it the camera intrinsic parameters by Cholesky factorization.

It would also be possible to propose non-linear algorithms using the characterization of $\mathrm{S}_{0}$ given in section 5 .

If the skew is zero then it is possible to identify a couple of intersecting orthogonal lines for each camera, namely those obtained by back-projecting the image points of coordinates $(1,0,0)$ and $(0,1,0)$. With these data it is possible to identify the calibration pencil by linear methods using twenty or more cameras [6].

Prospective applications of the concepts introduced in this paper include the analysis of critical motions for autocalibration of varying cameras with known skew and aspect ratio [4], [9]. Actually, after performing a projective calibration, it is equivalent to know the skew and aspect ratio of a camera and to know the lines in the virtual retinal plane through the optical center of the camera that intersect the absolute conic. Then, the Euclidean calibration is uniquely determined if and only if there is a single conic in space intersecting these set of lines.

\section{APPENDIX}

The following Lemma provides a method to identify as an $\alpha$-plane or as a $\beta$-plane a plane $\Pi \subset \Omega$.

Lemma 8.1. Let $\Pi \subset \Omega$ be a plane contained in the Klein quadric, and let us take three independent points of $\Pi$ of coordinates $r_{0}, r_{1}$ and $r_{2}$ with respect to some allowable coordinate system of $\mathbf{P}^{5}$. Let $M$ be the matrix of rows $r_{0}^{T}, r_{1}^{T}$ and $r_{2}^{T}$. Let us denote by $M_{i j k}$ the submatrix of $M$ given by the columns $i, j$ and $k$. There are two mutually excludent possibilities:

(1) $\operatorname{det} M_{123}=\operatorname{det} M_{246}=\operatorname{det} M_{145}=\operatorname{det} M_{356}=0$. In this case $\Pi$ is a $\beta$-plane.

(2) $\operatorname{det} M_{456}=\operatorname{det} M_{135}=\operatorname{det} M_{236}=\operatorname{det} M_{124}=0$. In this case $\Pi$ is a $\alpha$-plane.

Proof. The result follows from the consideration that only an $\alpha$-plane (resp. $\beta$ plane) intersects the four $\alpha$-planes (resp. $\beta$-planes) corresponding to the four vertices (resp. planes) of the reference tetrahedron of $\mathbf{P}^{3}$.

\section{ACKNOWLEDGEMENTS}

We want to thank F. Kahl for pointing us reference [6]. We also want to thank E. Arrondo for several useful conversations.

This work has been supported by the Comisión Interministerial de Ciencia y Tecnología (CICYT) of the Spanish Government under project TIC2001-3069.

\section{REFERENCES}

[1] D.A. Forsyth and J. Ponce, Computer Vision: A Modern Approach. Prentice Hall, New York, 2002.

[2] J. Harris, Algebraic Geometry. A first course. Graduate Texts in Mathematics 133, SpringerVerlag, New York, 1992.

[3] R.I. Hartley and A. Zisserman, Multiple View Geometry in Computer Vision. Cambridge University Press, Cambridge, UK, 2000. 
[4] F. Kahl, B. Triggs and K. Aastroom, Critical motions for auto-calibration when some intrinsic parameters can vary. Journal of Mathematical Imaging and Vision 13, 131-146, 2000.

[5] S.J. Maybank and O. Faugeras, A Theory of Self-Calibration of a Moving Camera. The International Journal of Computer Vision. 8, pp. 123-152, 1992.

[6] J. Ponce, On Computing Metric Upgrades of Projective Reconstructions Under the Rectangular Pixel Assumption. In Proc. of the SMILE 2000 Workshop on 3D Structure from Multiple Images of Large-Scale Environments, Springer-Verlag Lecture Notes in Computer Science 2018, pp. 52-67, 2000.

[7] J.G. Semple and G.T. Kneebone, Algebraic Projective Geometry. Oxford University Press, 1998.

[8] Y. Seo, A. Heyden, Auto-calibration from the orthogonality constraints, In International Conference on Computer Vision, 2000.

[9] P. Sturm, Critical motion sequences for monocular self-calibration and uncalibrated Euclidean reconstruction. In CVPR - IEEE International Conference on Computer Vision and Pattern Recognition, pp. 1100-1105, 1997.

[10] W. Triggs, Auto-calibration and the absolute quadric. In Proc. IEEE Conference on Computer Vision and Pattern Recognition, pages 609-614, 1997. 